\title{
Improved Effective Action for Light Quarks Beyond Chiral Limit
}

\author{
M. Musakhanov ${ }^{1}$ \\ Theoretical Physics Dept, Tashkent State University, Tashkent 700095, Uzbekistan \\ e-mail: yousuf@univer.tashkent.su,cc: yousuf@iaph.silk.org
}

\begin{abstract}
We propose an improvement of the Diakonov-Petrov Effective Action on the basis of the Lee-Bardeen results for the quark determinant in the instanton field. This Improved Effective Action provides proper account of the current quark masses, which is particularly important for strange quarks. This Action is successfully tested by the calculation of the quark condensate and by axial-anomaly low-energy theorems.
\end{abstract}

\section{Introduction}

Without any doubts instantons are a very important component of the QCD vacuum. Their properties are described by the average instanton size $\rho$ and interinstanton distance $R$. In 1982 Shuryak [1] fixed them phenomenologically as

$$
\rho=1 / 3 \mathrm{fm}, \quad R=1 \mathrm{fm} .
$$

From that time the validity of such parameters was confirmed by theoretical variational calculations [2] and recent lattice simulations of the QCD vacuum (see recent review $[3])$.

The presence of instantons in QCD vacuum very strongly affects light quark properties, owing to instanton-quark rescattering and consequent generation of quarkquark interactions.

These effects lead to the formation of the massive constituent interacting quarks. This implies spontaneous breaking of chiral symmetry (SBCS), which leads to the collective massless excitations of the QCD vaccum-pions. The most important degrees of freedom in low-energy QCD are these quasiparticles. So instantons play a leading role in the formation of the lightest hadrons and their interactions, while the confinment forces are rather unimportant, probably.

All of these properties are concentrated in the Effective Action in terms of quasiparticles. Effective actions for quarks in the field of the instantons go back to Shifman, Vainshtein, Zakharov [4]. A very successful attempt to construct this one was made by Diakonov and Petrov (DP) in 1986 (see recent paper[5] and recent detailed review [6] and references therein). Starting from the instanton model of QCD vacuum, they postulated the Effective Action on the basis of the interpolation formula for the well known expression for the light quark propagator $S_{ \pm}$in the field of the single instanton(antiinstanton):

$$
S_{ \pm} \approx S_{0}+\Phi_{ \pm, 0} \Phi_{ \pm, 0}^{\dagger} / i m
$$

\footnotetext{
${ }^{1}$ Associate Member of ICTP
} 
Here $S_{0}=(i \hat{\partial})^{-1}$ and $\Phi_{ \pm, 0}$ are the quark zero-modes generated by instantons ${ }^{2}$.

On the other hand, Lee\&Bardeen [7] (LB) derived the quark propagator in the background of many instantons in a much more sophisticated approximation than DP as

$S \approx S_{0}+\sum_{i}^{N}\left(S_{i}^{N Z M}-S_{0}\right)+\left(B^{-1}\right)_{i j}\left[\left|\Phi_{j, 0}>+R^{+}(-m)\right| \Phi_{j, 0}>\right]\left[<\Phi_{i, 0}\left|+<\Phi_{i, 0}\right| R(m)\right]$,

where

$$
B_{i j}=i m \delta_{i j}+a_{j i}
$$

and $a_{i j}$ is the overlapping matrix element of the quark zero-modes $\Phi_{ \pm, 0}$ generated by instantons(antiinstantons). This matrix element is nonzero only between instantons and antiinstantons (and vice versa) due to specific chiral properties of the zero-modes and equal to

$$
a_{-+}=-<\Phi_{-, 0}|i \hat{\partial}| \Phi_{+, 0}>.
$$

The overlapping of the quark zero-modes provides the propagating of the quarks by jumping from one instanton to another one. The quantity

$$
R(m)=\sum_{i}^{N} g\left(\hat{A}-\hat{A}_{i}\right)\left(S_{i}^{N Z M}-S_{0}\right)-i m\left[S_{0}+\sum_{i}^{N}\left(S_{i}^{N Z M}-S_{0}\right)\right],
$$

describes the contribution of the non-normalizable continuum. It was mentioned in [7] the importance of this contribution.

With this quark propagator (3) the corresponding fermionic determinant in the field of many instantons was calculated by LB, who found an amusing result for this quantity:

$$
\operatorname{det}_{N}=\operatorname{det} B, B_{i j}=i m \delta_{i j}+a_{j i},
$$

So, the determinant of the infinite matrix was reduced to the determinant of the finite matrix in the space of only zero-modes. From Eqs. (4), (5), (6) it is clear that for $N_{+} \neq N_{-}$

$$
\operatorname{det}_{N} \sim m^{\left|N_{+}-N_{-}\right|}
$$

which will strongly suppress the fluctuations of $\left|N_{+}-N_{-}\right|$. Therefore in final formulas we will assume $N_{+}=N_{-}=N / 2$.

Recently a correction to the result of Lee\&Bardeen in the simplest case of an instanton-anti-instanton molecule was proposed [12]. It was shown that the correction has a structure of the type

$$
\operatorname{det} B=\left(m^{2}\left(1+O\left(\rho^{6} R^{-6}\right)\right)+|a|^{2}\left(1+\frac{a+a^{*}}{|a|^{2}} O\left(\rho^{5} R^{-6}\right)\right)\right) .
$$

Since $a \sim \rho^{2} R^{-3}$, the second addend of the coefficient of $|a|^{2}$ in (8) is of order $O\left(\rho^{3} R^{-3}\right) \sim(1 / 3)^{3}$, therefore both correction terms which appear in that expression may be neglected. It follows that (6) properly take into account the masses of

\footnotetext{
${ }^{2} \Phi_{ \pm, \lambda}$ is the eigen-solution of the Dirac equation $\left(i \partial_{\mu}+g A_{ \pm, \mu}\right) \Phi_{ \pm, \lambda}=\lambda \Phi_{ \pm, \lambda}$ in the instanton(antiinstanton) field $A_{ \pm, \mu}\left(x ; \xi_{ \pm}\right)$.
} 
the current quarks. Here we observe the competition between current mass $m$ and overlapping matrix element $a \sim \rho^{2} R^{-3}$. With typical instanton sizes $\rho \sim 1 / 3 \mathrm{fm}$ and inter-instanton distances $R \sim 1 \mathrm{fm}, a$ is of the order of the strange current quark mass, $m_{s}=150 \mathrm{MeV}$. So in this case it is very important to take properly into account the current quark mass.

In our previous papers [8-10] we showed that the constituent quarks appear as effective degrees of freedom in the fermionic representation of $\operatorname{det} B$. This approach led to the DP Effective Action with a specific choice of these degrees of freedom. This Effective Action fulfilled some axial-anomaly low energy theorems in the chiral limit, but failed beyond this limit $[8,9]$. So this Action is hardly applicable to the strange quarks.

The aim of this work is to find an Improved Effective Action so as to take into account current quark masses. We essentially follow the same approach as in our previous works [8-10], but with another fermionic representation of $\operatorname{det} B$, which amounts to a different choice of the degrees of freedom in the Effective Action. This Improved Effective Action will be checked against direct calculations of the quark condensate and against axial-anomaly low energy theorems beyond the chiral limit.

\section{The Derivation of the Improved Effective Action}

The effective action follows from the fermionic representation of the $\operatorname{det}_{N}$ [5]. This is not a unique operation. The problem is to take a proper representation which will define the main degrees of freedom in low-energy QCD-constituent quarks.

Let us rewrite the $\operatorname{det}_{N}$ following the idea suggested in [11]. First, by introducing the Grassmanian $\left(N_{+}, N_{-}\right)$vector

$$
\Omega=\left(u_{1} \ldots u_{N_{+}}, v_{1} \ldots v_{N_{-}}\right)
$$

and

$$
\bar{\Omega}=\left(\bar{u}_{1} \ldots \bar{u}_{N_{+}}, \bar{v}_{1} \ldots \bar{v}_{N_{-}}\right)
$$

we can rewrite

$$
\operatorname{det}_{N}=\int d \Omega d \bar{\Omega} \exp (\bar{\Omega} B \Omega)
$$

where

$$
\bar{\Omega} B \Omega=\bar{\Omega}\left(i m+a^{T}\right) \Omega=i \sum_{+} m \bar{u}_{+} u_{+}+i \sum_{-} m \bar{v}_{-} v_{-}+\sum_{+-}\left(\bar{u}_{+} v_{-} a_{-+}+\bar{v}_{-} u_{+} a_{+-}\right)
$$

The product $\bar{u}_{+} v_{-} a_{-+}$and $\bar{u}_{+} i m u_{+}, \bar{v}_{-} i m v_{-}$can be rewritten in the form

$$
\begin{aligned}
\bar{u}_{+} a_{-+} v_{-}=-v_{-} a_{-+} \bar{u}_{+} & =-\left\langle(i \hat{\partial}+i m) \Phi_{-, 0} v_{-}\left|(i \hat{\partial}+i m)^{-1}\right|(i \hat{\partial}+i m) \Phi_{+, 0} \bar{u}_{+}\right\rangle . \\
\bar{u}_{+} i m u_{+} & =-\left\langle\Phi_{+, 0} u_{+}|(i \hat{\partial}+i m)| \Phi_{+, 0} \bar{u}_{+}\right\rangle \\
& =-\left\langle(i \hat{\partial}+i m) \Phi_{+, 0} u_{+}\left|(i \hat{\partial}+i m)^{-1}\right|(i \hat{\partial}+i m) \Phi_{+, 0} \bar{u}_{+}\right\rangle \\
\bar{v}_{-} i m v_{-} & =-\left\langle(i \hat{\partial}+i m) \Phi_{-, 0} v_{-}\left|(i \hat{\partial}+i m)^{-1}\right|(i \hat{\partial}+i m) \Phi_{-, 0} \bar{v}_{-}\right\rangle .
\end{aligned}
$$


The next step is to introduce $N_{+}, N_{-}$sources $\eta=\left(\eta_{+}, \eta_{-}\right)$and $N_{-}, N_{+}$sources $\bar{\eta}=$ $\left(\bar{\eta}_{-}, \bar{\eta}_{+}\right)$defined as:

$$
\begin{array}{ll}
\bar{\eta}_{-}=\left\langle(i \hat{\partial}+i m) \Phi_{-, 0} v_{-}\right| & , \quad \bar{\eta}_{+}=\left\langle(i \hat{\partial}+i m) \Phi_{+, 0} u_{+}\right| \\
\eta_{+}=\left|(i \hat{\partial}+i m) \Phi_{+, 0} \bar{u}_{+}\right\rangle & , \quad \eta_{-}=\left|(i \hat{\partial}+i m) \Phi_{-, 0} \bar{v}_{-}\right\rangle .
\end{array}
$$

Then $(\bar{\Omega} B \Omega)$ can be rewritten as

$$
(\bar{\Omega} B \Omega)=-\bar{\eta}_{+}(i \hat{\partial}+i m)^{-1} \eta_{+}-\bar{\eta}_{-}(i \hat{\partial}+i m)^{-1} \eta_{-}+\bar{\eta}_{-}(i \hat{\partial}+i m)^{-1} \eta_{+}+\bar{\eta}_{+}(i \hat{\partial}+i m)^{-1} \eta_{-}
$$

and $\operatorname{det}_{N}$ can be rewritten as

$$
\begin{aligned}
\operatorname{det}_{N} & =\int d \Omega d \bar{\Omega} \exp (\bar{\Omega} B \Omega) \\
& =(\operatorname{det}(i \hat{\partial}+i m))^{-1} \int d \Omega d \bar{\Omega} D \psi D \psi^{\dagger} \exp \int d x\left(\psi^{\dagger}(x)(i \hat{\partial}+i m) \psi(x)\right. \\
& \left.-\bar{\eta}_{+}(x) \psi(x)+\bar{\eta}_{-}(x) \psi(x)+\psi^{\dagger}(x) \eta_{+}(x)-\psi^{\dagger}(x) \eta_{-}(x)\right)
\end{aligned}
$$

The integration over Grassmanian variables $\Omega$ and $\bar{\Omega}$ (with the account of the $N_{f}$ flavors $\left.\operatorname{det}_{N}=\prod_{f} \operatorname{det} B_{f}\right)$ provides the fermionized representation of Lee\& Bardeen's result for $\operatorname{det}_{N}$ in the form:

$$
\begin{aligned}
& \operatorname{det}_{N}=\int D \psi D \psi^{\dagger} \exp \left(\int d^{4} x \sum_{f} \psi_{f}^{\dagger}\left(i \hat{\partial}+i m_{f}\right) \psi_{f}\right) \\
& \times \prod_{f}\left\{\prod_{+}^{N_{+}} V_{+}\left[\psi_{f}^{\dagger}, \psi_{f}\right] \prod_{-}^{N_{-}} V_{-}\left[\psi_{f}^{\dagger}, \psi_{f}\right]\right\},
\end{aligned}
$$

where

$V_{ \pm}\left[\psi_{f}^{\dagger}, \psi_{f}\right]=\int d^{4} x\left(\psi_{f}^{\dagger}(x)\left(i \hat{\partial}+i m_{f}\right) \Phi_{ \pm, 0}\left(x ; \xi_{ \pm}\right)\right) \int d^{4} y\left(\Phi_{ \pm, 0}^{\dagger}\left(y ; \xi_{ \pm}\right)\left(i \hat{\partial}+i m_{f}\right) \psi_{f}(y)\right)$.

Eq. (17) exactly represents the fermionic determinant in terms of constituent quarks $\psi_{f}$. This expression differs from the ansatz on the fixed $N$ partition function postulated by DP by another account of the current mass of quarks.

Let us calculate the quark propagator $S$ in the field of the instanton-anti-instanton pairs. First, we calculate partition function $Z_{N}$

$$
Z_{N}=\operatorname{det}_{N}=-m^{2}-|a|^{2}
$$

where

$$
a=\left\langle\Phi_{-, 0}|i \hat{\partial}| \Phi_{+, 0}\right\rangle .
$$

Taking into account (19) and (17), we find the propagator

$$
S=(i \hat{\partial}+i m)^{-1}-\frac{i m\left(\Phi_{+, 0} \Phi_{+, 0}^{\dagger}+\Phi_{-, 0} \Phi_{-, 0}^{\dagger}\right)+a \Phi_{-, 0} \Phi_{+, 0}^{\dagger}+a^{*} \Phi_{+, 0} \Phi_{-, 0}^{\dagger}}{m^{2}+|a|^{2}} .
$$

As was mentioned before, with typical instanton sizes $\rho$ and inter-instanton distances $R(1), a$ is of the order of the strange current quark mass, $m_{s}=150 \mathrm{MeV}$. Again we 
conclude that, in this case it is very important to take into account the current quark mass.

Keeping in mind the low density of the instanton media, which allows independent averaging over positions and orientations of the instantons, Eq. (17) leads to the partition function

$$
Z_{N}=\int D \psi D \psi^{\dagger} \exp \left(\int d^{4} x \sum_{f} \psi_{f}^{\dagger}\left(i \hat{\partial}+i m_{f}\right) \psi_{f}\right) W_{+}^{N_{+}} W_{-}^{N_{-}}
$$

where

$$
W_{ \pm}=\int d \xi_{ \pm} \prod_{f} V_{ \pm}\left[\psi_{f}^{\dagger} \psi_{f}\right]
$$

Developing the integral in $\xi_{ \pm}$, we get

$$
\begin{aligned}
& \int d \xi_{ \pm}(i \hat{\partial}+i m) \Phi_{ \pm}(x) \Phi_{ \pm}^{\dagger}(y)(-i \overleftarrow{\hat{\partial}}+i m) \\
& =\int d^{4} z e^{i\left(k_{2}-k_{1}\right) z} \frac{d^{4} k_{1}}{(2 \pi)^{4}} \frac{d^{4} k_{2}}{(2 \pi)^{4}} e^{i k_{1} x} e^{-i k_{2} y} N_{c}^{-1} \phi^{\prime}\left(k_{1}\right)\left|k_{1}\right| \phi^{\prime}\left(k_{2}\right)\left|k_{2}\right|\left(\frac{1 \pm \gamma_{5}}{2}\right. \\
& \left.+\quad \frac{i m \hat{k}_{2}}{k_{2}^{2}} \frac{1 \mp \gamma_{5}}{2}-\frac{i m \hat{k}_{1}}{k_{1}^{2}} \frac{1 \pm \gamma_{5}}{2}+\frac{m^{2} \hat{k}_{1} \hat{k}_{2}}{k_{1}^{2} k_{2}^{2}} \frac{1 \mp \gamma_{5}}{2}\right),
\end{aligned}
$$

whence

$$
\left.W_{ \pm}=(-i)^{N_{f}}\left(\frac{4 \pi^{2} \rho^{2}}{N_{c}}\right)^{N_{f}} \int \frac{d^{4} z}{V} \operatorname{det}_{f}\left(i J_{ \pm}(z)\right)\right)
$$

where we have set

$$
\begin{aligned}
J_{ \pm}(z)_{f g} & =\int \frac{d^{4} k_{1} d^{4} k_{2}}{(2 \pi)^{8}} e^{\left.i\left(k_{2}-k_{1}\right) z\right)}\left|k_{1}\right| \phi^{\prime}\left(k_{1}\right)\left|k_{2}\right| \phi^{\prime}\left(k_{2}\right) \psi_{f}^{\dagger}\left(k_{1}\right)\left(\frac{1 \pm \gamma_{5}}{2}\right. \\
& \left.+\frac{i m \hat{k}_{2}}{k_{2}^{2}} \frac{1 \mp \gamma_{5}}{2}-\frac{i m \hat{k}_{1}}{k_{1}^{2}} \frac{1 \pm \gamma_{5}}{2}+\frac{m^{2} \hat{k}_{1} \hat{k}_{2}}{k_{1}^{2} k_{2}^{2}} \frac{1 \mp \gamma_{5}}{2}\right) \psi_{g}\left(k_{2}\right)
\end{aligned}
$$

The two remarkable formulas

$$
(a b)^{N}=\int d \lambda \exp \left(N \ln \frac{a N}{\lambda}-N+\lambda b\right)(N>>1) .
$$

and

$$
\exp (\lambda \operatorname{det}[i A])=\int d \Phi \exp \left[-\left(N_{f}-1\right) \lambda^{-\frac{1}{N_{f}-1}}(\operatorname{det} \Phi)^{\frac{1}{N_{f}-1}}+i \operatorname{tr}(\Phi A)\right]
$$

have been used here. It is possible to check these formulas by the saddle-point approximation of the integrals. They were proposed in [5] and we followed this approach. Formula (27) leads to exponentiation, while (28) leads to the bosonization of the partition function (22). Starting from these formulas, we find

$$
Z_{N}=\int d \lambda_{+} d \lambda_{-} D \Phi_{+} D \Phi_{-} \exp \left(-W\left[\lambda_{+}, \Phi_{+} ; \lambda_{-}, \Phi_{-}\right]\right)
$$


where

$$
\begin{aligned}
W\left[\lambda_{+}, \Phi_{+} ; \lambda_{-}, \Phi_{-}\right] & =-\sum_{ \pm}\left(N_{ \pm} \ln \left[\left(\frac{4 \pi^{2} \rho^{2}}{N_{c}}\right)^{N_{f}} \frac{V \lambda_{ \pm}}{N_{ \pm}}\right]-N_{ \pm}\right)+w_{\Phi}+w_{\psi}, \\
w_{\Phi} & =\int d^{4} x \sum_{ \pm}\left(N_{f}-1\right) \lambda_{ \pm}^{-\frac{1}{N_{f}-1}}\left(\operatorname{det} \Phi_{ \pm}\right)^{\frac{1}{N_{f}-1}} \\
w_{\psi} & =-\operatorname{Tr} \ln \left(-\hat{k}+i m_{f}+i\left|k_{1}\right| \phi^{\prime}\left(k_{1}\right)\left|k_{2}\right| \phi^{\prime}\left(k_{2}\right) \sum_{ \pm} \Phi_{ \pm}\left(k_{1}-k_{2}\right)\left(\frac{1 \pm \gamma_{5}}{2}\right.\right. \\
& \left.\left.+\frac{i m \hat{k}_{2}}{k_{2}^{2}} \frac{1 \mp \gamma_{5}}{2}-\frac{i m \hat{k}_{1}}{k_{1}^{2}} \frac{1 \pm \gamma_{5}}{2}+\frac{m^{2} \hat{k}_{1} \hat{k}_{2}}{k_{1}^{2} k_{2}^{2}} \frac{1 \mp \gamma_{5}}{2}\right)\left(-\hat{k}+i m_{f}\right)^{-1}\right) .
\end{aligned}
$$

Variation of the total action $W\left[\lambda_{+}, \Phi_{+} ; \lambda_{-} \Phi_{-}\right]$over $\lambda_{ \pm}, \Phi_{ \pm}$must vanish in the common saddle-point. In this point

$$
\lambda_{ \pm}=\lambda, \Phi_{ \pm, f g}=\Phi_{ \pm, f g}(0)=\Phi_{f} \delta_{f g} .
$$

This condition leads to the definition of the constituent mass $M_{f}$ and the form-factor $F\left(k^{2}\right)$, i. e.,

$$
M_{f}(k)=M_{f} F^{2}(k)=\lambda k^{2} \phi^{\prime 2}(k) \Phi_{f} .
$$

The contribution of the quark loop to the saddle-point equation is

$$
\operatorname{Tr} \ln \left[\left(-\hat{k}+i m+i k^{2} \phi^{\prime 2} \sum_{ \pm} \Phi_{ \pm}\left(\frac{1 \pm \gamma_{5}}{2}+\frac{m^{2}}{k^{2}} \frac{1 \mp \gamma_{5}}{2} \pm i m \hat{k} \gamma_{5}\right)\right)(-\hat{k}+i m)^{-1}\right]
$$

The details of the calculations of the $\operatorname{Tr} \ln$ are

$$
\begin{array}{r}
\operatorname{Tr}\left[\ln \left(-\hat{k}\left(1+\alpha \gamma_{5}\right)+i\left(\Lambda_{+} \frac{1+\gamma_{5}}{2}+\Lambda_{-} \frac{1-\gamma_{5}}{2}\right) /(-\hat{k})\right]=\right. \\
2 \ln \left(1-\alpha^{2}+\frac{\Lambda_{+} \Lambda_{-}}{k^{2}}\right),
\end{array}
$$

where

$$
\begin{gathered}
\alpha=\phi^{\prime 2} m\left(\Phi_{+}-\Phi_{-}\right), \\
\Lambda_{ \pm}=m+k^{2} \phi^{\prime 2}\left(\Phi_{ \pm}+\Phi_{\mp} \frac{m^{2}}{k^{2}}\right) .
\end{gathered}
$$

Finally, the saddle-point equation is

$$
\frac{4 V N_{c}}{N} \int \frac{d^{4} k}{(2 \pi)^{4}} \frac{M_{f}^{2}(k)+m_{f} M_{f}(k)+2 M_{f}^{2}(k) \frac{m_{f}^{2}}{k^{2}}}{k^{2}+M_{f}^{2}(k)+2 m_{f} M_{f}(k)+2 M_{f}^{2}(k) \frac{m_{f}^{2}}{k^{2}}}=1 .
$$

We keep only $O\left(m_{f}\right)$ terms and define $M_{f}=M_{0}+\gamma m_{f}$. By expanding of the left side of saddle-point equation in $m_{f}$, we get

$$
\begin{array}{r}
\frac{4 V N_{c}}{N} \int \frac{d^{4} k}{(2 \pi)^{4}} \frac{M_{0}^{2}(k)}{k^{2}+M_{0}^{2}(k)}=1, \\
2 \gamma \int k^{2} d k^{2} \frac{k^{2} F^{2}}{\left(k^{2}+M_{0}^{2} F^{4}\right)^{2}}=\int k^{2} d k^{2} \frac{\left(M_{0}^{2} F^{4}-k^{2}\right) F^{2}}{\left(k^{2}+M_{0}^{2} F^{4}\right)^{2}} .
\end{array}
$$


The form-factor $F$ is related to the zero-mode wave function in momentum space $\Phi_{ \pm}\left(k ; \xi_{ \pm}\right)$and is equal to

$$
F\left(k^{2}\right)=-t \frac{d}{d t}\left[I_{0}(t) K_{0}(t)-I_{1}(t) K_{1}(t)\right] \quad, \quad t=\frac{1}{2} \sqrt{k^{2}} \rho .
$$

In [13] it was proposed the simplified expression

$$
F=\frac{\Lambda^{2}}{\Lambda^{2}+k^{2}}
$$

where $\Lambda^{2} \sim 2 / \rho^{2}=0.72 G e V^{2}$.

The solution of the saddle-point equation (35) corresponding $M_{0}=340 \mathrm{MeV}$ demands $\Lambda^{2}=0.76 \mathrm{GeV}^{2}$. At $\Lambda^{2}=0.76 \mathrm{GeV}^{2} \gamma=-0.54$.

Finally, the consituent quark propagator has a form:

$$
S=\left(i \hat{\partial}+i\left(m_{f}+M_{f} F^{2}\right)\right)^{-1}
$$

where $M_{f}$ and $F$ are given by (35), (36), (38).

\section{DP Effective Action}

In order to clarify the important differences between Improved and DP Effective Actions we provide below the derivation of DP Effective Action and propagator without giving all the details .

In order to find the DP Effective Action, we must start from the following representation of $\exp \left(\bar{\Omega} a^{T} \Omega\right)$ :

$$
\begin{aligned}
& \exp \left(\bar{\Omega} a^{T} \Omega\right) \\
& =\exp \int\left(-\bar{\eta}(i \hat{\partial})^{-1} \eta\right) \\
& =(\operatorname{det}(i \hat{\partial}))^{-1} \int D \psi D \psi^{\dagger} \exp \int d x\left(\psi^{\dagger}(x) i \hat{\partial} \psi(x)-\bar{\eta}(x) \psi(x)+\psi^{\dagger}(x) \eta(x)\right),
\end{aligned}
$$

where $N_{+}, N_{-}$sources $\eta=\left(\eta_{+}, \eta_{-}\right)$and $N_{-}, N_{+}$sources $\bar{\eta}=\left(\bar{\eta}_{-}, \bar{\eta}_{+}\right)$defined as:

$$
\begin{aligned}
& \bar{\eta}_{-}=<i \hat{\partial} \Phi_{-, 0} v_{-}\left|, \quad \bar{\eta}_{+}=<i \hat{\partial} \Phi_{+, 0} u_{+}\right| \\
& \eta_{+}=\left|i \hat{\partial} \Phi_{+, 0} \bar{u}_{+}>, \quad \eta_{-}=\right| i \hat{\partial} \Phi_{-, 0} \bar{v}_{-}>
\end{aligned}
$$

As shown in $[8,10]$, this leads to:

$$
\begin{aligned}
& \operatorname{det}_{N}=\int D \psi D \psi^{\dagger} \exp \left(\int d^{4} x \sum_{f} \psi_{f}^{\dagger} i \hat{\partial} \psi_{f}\right) \\
& \times \prod_{f}\left\{\prod_{+}^{N_{+}}\left(i m_{f}+V_{+}^{\prime}\left[\psi_{f}^{\dagger}, \psi_{f}\right]\right) \prod_{-}^{N_{-}}\left(i m_{f}+V_{-}^{\prime}\left[\psi_{f}^{\dagger}, \psi_{f}\right]\right)\right\},
\end{aligned}
$$

where

$$
V_{ \pm}^{\prime}\left[\psi_{f}^{\dagger}, \psi_{f}\right]=\int d^{4} x\left(\psi_{f}^{\dagger}(x) i \hat{\partial} \Phi_{ \pm, 0}\left(x ; \xi_{ \pm}\right)\right) \int d^{4} y\left(\Phi_{ \pm, 0}^{\dagger}\left(y ; \xi_{ \pm}\right) i \hat{\partial} \psi_{f}(y)\right)
$$


Eq. (42) coincides with the ansatz for the fixed $N$ partition function postulated by DP, except for the unessential sign in front of $V_{ \pm}^{\prime}$. This partition function leads to the quit different equations for the effective mass $M_{f}^{D P}$ and the propagator than (35), (36) and (39).

Effective mass $M_{f}^{D P}$ now obey the equation:

$$
4 N_{c} V \int \frac{d^{4} k}{(2 \pi)^{4}} \frac{M_{f}^{2} F^{4}\left(k^{2}\right)}{M_{f}^{2} F^{4}\left(k^{2}\right)+k^{2}}=N+\frac{m_{f} M_{f} V N_{c}}{2 \pi^{2} \rho^{2}},
$$

being imposed, which also describes the shift of the effective mass of the quark $M_{f}^{D P}$ due to current mass $m_{f}$. Expanding (44) with respect to $m_{f}$ yields

$$
M_{f}^{D P}=M_{0}+\gamma_{D P} m_{f},
$$

where

$$
\gamma_{D P}^{-1}=\rho^{2} \int_{0}^{\infty} d k^{2} \frac{k^{4} F^{4}\left(k^{2}\right)}{\left(M_{0}^{2} F^{4}\left(k^{2}\right)+k^{2}\right)^{2}} .
$$

Such integrals converge due to the form factor $F\left(k^{2}\right)$. Assuming for the parameters $\rho$ and $R$ the values (1) - which correspond to $M_{0}=340 \mathrm{MeV}$ - we find

$$
\gamma_{D P}=2.4
$$

Also, from DP Effective Action constituent quark propagator has a form:

$$
\left.S^{D P}=\left(i \hat{\partial}+M_{f}^{D P} F^{2}\right)\right)^{-1},
$$

where $M_{f}^{D P}$ is given by (45), (46).

\section{Tests for the Improved Effective Action}

Improved and DP Effective Actions coincides in chiral limit. So, we may expect essential differences in the results only beyond this limit.

We will test (30) by calculating the quark condensate and axial-anomaly lowenergy theorems, which will be reduced to the calculations of the specific correlators.

For these calculations we need an Improved Effective Action in the presence of an external electromagnetic field $a$ and of a field $\kappa$ coupled with the topological density $g^{2} G \tilde{G}$. A similar problem was solved in our previous works $[9,10]$ and we follow this method. The most essential part of the Improved Effective Action, $w_{\psi}$, is transformed to

$$
\begin{aligned}
w_{\psi}[a, \kappa] & =-\sum_{f} \operatorname{Tr} \ln \left(-\hat{K}+i m_{f}+i F\left(k_{1}\right) F\left(k_{2}\right) M_{f} \sum_{ \pm} \phi_{ \pm}(1 \pm(\kappa f))^{N_{f}^{-1}}\left(\frac{1 \pm \gamma_{5}}{2}\right.\right. \\
& \left.\left.\left.+\frac{i m \hat{k}_{2}}{k_{2}^{2}} \frac{1 \mp \gamma_{5}}{2}-\frac{i m \hat{k}_{1}}{k_{1}^{2}} \frac{1 \pm \gamma_{5}}{2}+\frac{m^{2} \hat{k}_{1} \hat{k}_{2}}{k_{1}^{2} k_{2}^{2}} \frac{1 \mp \gamma_{5}}{2}\right)\right)\left(-\hat{k}+i m_{f}\right)^{-1}\right)
\end{aligned}
$$

where normalization is $\phi_{ \pm}(k=0)=1$ and gauged momentum is $\hat{K}=\hat{k}-e Q \hat{a}$ as usual.

The quark condensate from Improved Effective Action. 


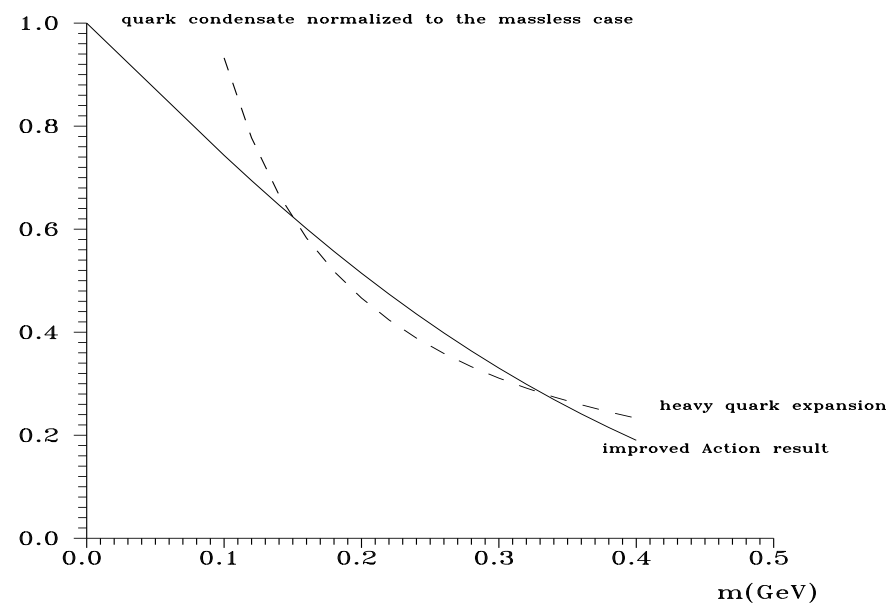

Figure 1: The dependence of the condensate $i\left\langle\psi^{\dagger} \psi\right\rangle$ on the current quark mass $m$ normalized to the massless case. The solid line represents the result of the calculations with Improved Effective Action, Eq.(52). The dashed line represents the formula (54) for the heavy quark condensate.

First, we calculate the quark condensate by using the evident formula

$$
\begin{aligned}
i<\psi^{\dagger} \psi> & =Z_{N}^{-1} \frac{\partial Z_{N}}{\partial m} \\
& =-\frac{\delta W}{\delta m_{f}}=-\left.\sum_{ \pm}\left(\frac{\delta w_{\Phi}}{\delta \Phi_{ \pm}}+\frac{\delta w_{\psi}}{\delta \Phi_{ \pm}}\right)\right|_{\Phi} \frac{\delta \Phi}{\delta m_{f}} \\
& +\operatorname{Tr} i\left[\left(-\hat{k}+i m_{f}+i F^{2} M_{f}\right)^{-1}-\left(-\hat{k}+i m_{f}\right)^{-1}\right]
\end{aligned}
$$

Another way is to calculate it directly

$$
i<\psi^{\dagger} \psi>=\operatorname{Tr} i\left[\left(-\hat{k}+i m_{f}+i F^{2} M_{f}\right)^{-1}-\left(-\hat{k}+i m_{f}\right)^{-1}\right] .
$$

The first term in (50) vanishes at the saddle-point and we have a perfect equivalence of the two calculations of the condensate, in contrast with analogous calculations with the DP Action[5,9](see also below). With the formula (51) we get

$$
i<\psi_{f}^{\dagger} \psi_{f}>=N_{c} \int \frac{k^{2} d k^{2}}{4 \pi^{2}}\left(\frac{m_{f}+M_{f} F^{2}\left(k^{2}\right)}{k^{2}+\left(m_{f}+M_{f} F^{2}\left(k^{2}\right)\right)^{2}}-\frac{m_{f}}{x+m_{f}^{2}}\right) .
$$

Simple numerical calculations by using (35), (36), (38) leads to the

$$
\begin{aligned}
i<\psi^{\dagger} \psi>\left.\right|_{m=0} & =0.0171 \mathrm{GeV}^{3}, \\
i<\psi^{\dagger} \psi>\left.\right|_{m=0.15 G e V} & =0.0107 \mathrm{GeV}^{3}, \\
\frac{<\psi^{\dagger} \psi>\left.\right|_{m=0.15}}{<\psi^{\dagger} \psi>\left.\right|_{m=0}}-1 & =-0.37 .
\end{aligned}
$$

More detailed information on the dependence of the condensate on the current quark mass is presented in Fig.1. The solid line represents the result of the calculations of Eq.(52). It is clear from the figure that the calculations with Improved Effective Action 
lead to the expected dependence on the current mass. The dashed line represents the formula for the heavy quark condensate:

$$
\begin{aligned}
i<\psi^{\dagger} \psi> & =\frac{g^{2}}{2^{4} \pi^{2} 3 m V} \int d x G_{\mu \nu}^{a} G_{\mu \nu}^{a} \\
& =\frac{2 N}{3 m V}=\frac{2}{3 m R^{4}}=0.0016 m^{-1}
\end{aligned}
$$

This formula was derived in the lowest second order expansion over $G / m^{2}[15]$. It is interesting that both curves (normalized to the massless case) almost coincide in the region of $m \sim 0.3 \mathrm{GeV}$.

The quark condensate from DP Effective Action.

Two ways of the calculation of the quark condensate mentioned above lead to quit different formulas in case of DP Effective Action as

$$
\frac{1}{V Z_{N}^{D P}} \frac{d Z_{N}^{D P}}{d m_{f}}=\frac{N_{c} M_{f}^{D P}}{2 \pi^{2} \rho^{2}}
$$

and

$$
i<\psi_{f}^{\dagger} \psi_{f}>=4 N_{c} \int \frac{d^{4} k}{(2 \pi)^{4}} \frac{M_{f}^{D P} F^{2}\left(k^{2}\right)}{M_{f}^{D P 2} F^{4}\left(k^{2}\right)+k^{2}}
$$

In the chiral limit the formulas (52) and (56) coincide with each other and give the value which is almost the same as given by (55). The current mass dependence of the quark condensate is induced in case of DP Effective Action by the $m_{f}$-dependence of the effective mass $M_{f}^{D P}$ which is given by (45), (46). From (55) we get

$$
\frac{1}{V Z_{N}^{D P}} \frac{d Z_{N}^{D P}}{d m}=(0.018+0.045 m) G e V^{3}
$$

and from (56)

$$
i<\psi^{\dagger} \psi>=(0.016+0.181 m) G e V^{3}
$$

Therefore, both formulas (57) and (58), derived from DP Effective Action, give completely wrong (and different) dependence on $m$ beoynd chiral limit.

The next test is related to axial-anomaly low-energy theorems $(L E T)[14]$. These theorems were used in $[8,9]$ to check DP Effective Action. The DP Effective Action was able to reproduce LET only in chiral limit and failed beyond this limit.

\section{$\underline{L E T 1}$}

Nonvanishing of the $\eta^{\prime}$ meson mass $m_{\eta^{\prime}}$ even in chiral limit (due to axial anomaly) implies that for real photons the matrix element of the divergence of the singlet axial current vanishes in the $q^{2}<<m_{\eta^{\prime}}^{2}$ limit, giving rise to the following low energy theorem (LET1):

$$
\left\langle 0\left|N_{f} \frac{g^{2}}{16 \pi^{2}} G \tilde{G}\right| 2 \gamma\right\rangle+2 i \sum_{f} m_{f}\left\langle 0\left|\psi_{f}^{\dagger} \gamma_{5} \psi_{f}\right| 2 \gamma\right\rangle=N_{c} \frac{e^{2}}{4 \pi^{2}} \sum_{f} Q_{f}^{2} F^{(1)} \tilde{F}^{(2)},
$$


where $F_{\mu \nu}^{(i)}=\epsilon_{i, \mu} q_{i, \nu}-\epsilon_{i, \nu} q_{i, \mu}$ and $q_{i}, \epsilon_{i}(i=1,2)$ are the momentum and polarization vectors of photons and $q=q_{1}+q_{2}$ respectively. Eq. (59) is an exact low energy relation, which cannot be fulfilled in the framework of perturbation theory. Only a nonperturbative contribution of order $g^{-2}$ - as the one provided by instantons - may cancel the factor $g^{2}$ at the first term of the l.h.s.. The first term of the 1.h.s. in (59) is calculated from three-point correlator of the operator $g^{2} G \tilde{G}$ and two operators of the electromagnetic currents. It is clear from (49) that this correlator is equal to

$$
\left.\frac{\delta^{3} w_{\psi}[a, \kappa]}{\delta \kappa \delta a \delta a}\right|_{\phi=1, a, \kappa=0}
$$

The operator $g^{2} G \tilde{G}$ generates the vertex if $F^{2} M_{f} N_{f}^{-1} \gamma_{5}$, where $f\left(q^{2}\right)$ is a momentum representation of the instanton contribution in the operator $g^{2} G \tilde{G}(x)$ and $f(0)=32 \pi^{2}$. At small $q^{2}$ this vertex is reduces to

$$
32 \pi^{2} i F^{2} M_{f} N_{f}^{-1} \gamma_{5}
$$

Then, the three-angular diagrams corresponding to the the anomaly contribution (the first term in the l.h.s. of (59)), with vertices (61), $e Q_{f} \gamma_{\mu}$ and propagator (39) leads to

$$
2 i N_{c} e^{2} Q_{f}^{2} F^{(1)} \tilde{F}^{(2)} \Gamma_{f},
$$

where $\Gamma_{f}$, the factor coming from the diagram of the process considered, may be calculated analytically if we approximate the form factor $F$ by 1 . In this approximation

$$
\Gamma_{f}=\frac{M_{f}}{8 \pi^{2}\left(M_{f}+m_{f}\right)}
$$

In the same approximation the current mass contribution (the second term in the l.h.s. of $(59))$ leads to

$$
2 i N_{c} e^{2} Q_{f}^{2} F^{(1)} \tilde{F}^{(2)} \frac{m_{f}}{8 \pi^{2}\left(M_{f}+m_{f}\right)}
$$

At the next step we combine (62) and (64) and sum up over flavors. As a result, the l.h.s. and the r.h.s of eq. (59) coincide with each other. So, Improved Effective Action immediately fulfills low energy-theorem (59) even beyond the chiral limit in contrast with DP Effective Action result [9].

If we take into account the form factor $F$ in (62), (64) and give the model parameters the values (1), we find[8] a variation of $\sim 17 \%$.

\section{LET2, LET3}

Further tests for Improved Effective Action can be obtained from the matrix elements of the divergence of the singlet axial current between vacuum and meson states. Neglecting $O\left(\mathrm{~m}^{2}\right)$ terms, we get the following equations:

$$
\begin{aligned}
\left\langle 0\left|N_{f} \frac{g^{2}}{16 \pi^{2}} G \tilde{G}\right| \eta\right\rangle & =-2 i m_{s}\left\langle 0\left|\psi_{s}^{\dagger} \gamma_{5} \psi_{s}\right| \eta\right\rangle \\
\left\langle 0\left|N_{f} \frac{g^{2}}{16 \pi^{2}} G \tilde{G}\right| \pi^{0}\right\rangle & =-i\left(m_{u}-m_{d}\right)\left\langle 0\left|\psi^{\dagger} \tau_{3} \gamma_{5} \psi\right| \pi^{0}\right\rangle,
\end{aligned}
$$


which we call LET2 and LET3 respectively. These matrix elements are reduced to two-point correlators. It is rather easy to show that Improved Effective Action satisifies LET2 (65) and LET3 (66).

From previous considerations it follows that the factor $g^{2} G \tilde{G}$ generates the vertex $i M f F^{2} \gamma_{5} N_{f}^{-1}$ and the $\eta$-meson gives rise to $i M_{s} \lambda_{8} F^{2} \gamma_{5}$. The structure of the mass matrix $M$ is

$$
M=M_{0}+\gamma\left(m_{s}\left(\frac{1}{3}-\frac{1}{\sqrt{ } 3} \lambda_{8}\right)+m_{u} \frac{1+\tau_{3}}{2}+m_{d} \frac{1-\tau_{3}}{2}\right) .
$$

Then at small $q$ (and neglecting $\left.m_{u, d}\right)$

$$
\begin{aligned}
\left\langle 0\left|N_{f} \frac{g^{2}}{16 \pi^{2}} G \tilde{G}\right| \eta\right\rangle & =-\frac{16 N_{c}}{\sqrt{ } 3} \int \frac{d^{4} k}{(2 \pi)^{4}} F^{4}\left(k^{2}\right)\left[\frac{M_{s}^{2}}{\left(\left(M_{s} F^{2}\left(k^{2}\right)+m_{s}\right)^{2}+k^{2}\right)^{2}}\right. \\
& \left.-\frac{M_{0}^{2}}{\left(M_{0}^{2} F^{4}\left(k^{2}\right)+k^{2}\right)^{2}}\right] .
\end{aligned}
$$

Expanding (68) over $m_{s}$, we get

$$
\left\langle 0\left|N_{f} \frac{g^{2}}{16 \pi^{2}} G \tilde{G}\right| \eta\right\rangle=-\frac{16 N_{c} m_{s}}{\sqrt{ } 3} \int \frac{d^{4} k}{(2 \pi)^{4}} F^{4}\left(k^{2}\right) M_{0} \frac{2 \gamma k^{2}-2 M_{0}^{2} F^{2}\left(k^{2}\right)}{\left(k^{2}+M_{0}^{2} F^{4}\left(k^{2}\right)\right)^{2}} .
$$

From eq. (36) for the $\gamma$-factor we find that

$$
2 \gamma \int \frac{d^{4} k}{(2 \pi)^{4}} \frac{k^{2} F^{4}}{\left(k^{2}+M_{0}^{2} F^{4}\right)^{2}} \approx \int \frac{d^{4} k}{(2 \pi)^{4}} \frac{\left(M_{0}^{2} F^{4}-k^{2}\right) F^{2}}{\left(k^{2}+M_{0}^{2} F^{4}\right)^{2}}
$$

It is clear now that by using (70) the l.h.s of (65) is reduced to the r.h.s. of (65), which is equal to

$$
-2 i m_{s}\left\langle 0\left|\psi_{s}^{\dagger} \gamma_{5} \psi_{s}\right| \eta\right\rangle=\frac{16 N_{c} m_{s}}{\sqrt{ } 3} \int \frac{d^{4} k}{(2 \pi)^{4}} \frac{M_{0} F^{2}}{k^{2}+M_{0}^{2} F^{4}} .
$$

The calculations with LET3 (66) are almost the same as with LET2 (65). Again, by using (70) l.h.s. and r.h.s. of (66) coincide with each other. Hence Improved Effective Action satisfies LET2 and LET3, (65) and (66) respectively. For comparison, DP Effective Action failed to reproduce these LET2 and LET3 [9].

Therefore, Improved Effective Action generates correct current mass dependence of the vacuum quark condensate, satisfies low-energy theorems LET2, LET3 for the two-point correlators (65) and (66) respectively and also satisfies LET1 for the threepoint correlator (59) even beyond chiral limit. We conclude that our model works properly beyond chiral limit and provides the background for taking into account strange quarks.

This work was initiated during my visit RCNP, Osaka University. I am very grateful to H. Toki and F.Araki for the useful discussions at the early stage of this work and E. Di Salvo for the comments and suggestions. I acknowledge a partial support by the grant INTAS-96-0597ext and by the grant 11/97 of the State Committee for Science and Technology of Uzbekistan. 


\section{References}

[1] E. V. Shuryak, Nucl. Phys. B 203, 93, 116 (1982)

[2] D. Diakonov and V. Petrov, Nucl. Phys. B 245, 259 (1984)

[3] T. De Grand, A. Hasenfratz and T. Kovacs, in Proceedings of 1997 Yukawa International Seminar "Non-perturbative QCD. Structure of the QCD Vacuum", Kyoto, 1997, edited by K-I.Aoki, O.Miyamura and T.Suzuki (Progress of Theoretical Physics Supplement, 131, 1998), p. 573

[4] M.A. Shifman, A.I. Vainshtein, V.I. Zakharov, Nucl. Phys. B 166, 493 (1980)

[5] D.I. Diakonov, M.V. Polyakov, C. Weiss, Nucl. Phys. B 461, 539 (1996)

[6] T. Schaefer and E. Shuryak, hep-ph/9610451, Rev. Mod. Phys. 70, 323 (1998)

[7] C. Lee, W. A. Bardeen, Nucl. Phys. B 153, 210 (1979)

[8] M. M. Musakhanov, F. C. Khanna, Phys. Lett. B 395, 298 (1997)

[9] E. Di Salvo, M.M. Musakhanov, hep-ph/9706537, Europ. Phys. J. C 5, 501 (1998)

[10] F. Araki, M. Musakhanov, H. Toki, hep-ph/9808290

[11] V.F. Tokarev, preprint INP P-0406, Moscow 1985; Soviet J. Teor. Math. Phys., 73, 223 (1987)

[12] A.G. Zubkov, O.V. Dubasov, B.O. Kerbikov, hep-ph/9712549

[13] V.Yu. Petrov, M.V. Polyakov, R. Ruskov, C. Weiss and K. Goeke, hep-ph/9807229

[14] M.A. Shifman, Sov. Phys. Usp. 32, 289 (1989)

[15] A.I. Vainshtein, V.I. Zakharov, V.A. Novikov, M.A. Shifman, Sov.J.Nucl.Phys. 39, 77 (1984). 